\title{
Frequency Tuning Characteristics of a THz-wave Parametric Oscillator
}

\author{
Zhongyang $\mathrm{Li}^{1 *}$, Pibin Bing ${ }^{1}$, Degang $\mathrm{Xu}^{2}$, and Jianquan $\mathrm{Yao}^{2}$ \\ ${ }^{1}$ North China University of Water Resources and Electric Power, Zhengzhou 450011, China \\ ${ }^{2}$ College of Precision Instrument and Opto-electronics Engineering, Institute of Laser and \\ Opto-electronics, Tianjin University, Tianjin 300072, China
}

(Received October 9, 2012 : revised December 14, 2012 : accepted December 27, 2012)

\begin{abstract}
Frequency tuning characteristics of a THz-wave by varying phase-matching angle and pump wavelength in a noncollinear phase-matching THz-wave parametric oscillator (TPO) are analyzed. A novel scheme to realize the tuning of a THz-wave by moving the cavity mirror forwards and backwards is proposed in a noncollinear phase-matching TPO. The parametric gain coefficients of the THz-wave in a $\mathrm{LiNbO}_{3}$ crystal are explored under different working temperatures. The relationship between the poling period of periodically poled $\mathrm{LiNbO}_{3}$ (PPLN) and the THz-wave frequency under the condition of a quasi-phasematching configuration is deduced. Such analyses have an impact on the experiments of the TPO.
\end{abstract}

\footnotetext{
Keywords : THz-wave parametric oscillator, Noncollinear phase-matching, Quasi-phase-matching, Tuning THz-wave

OCIS codes : (190.4410) Nonlinear optics, parametric process; (190.4970) Parametric oscillator and amplifiers; (140.3070) Infrared and far-infrared lasers
}

\section{INTRODUCTION}

Applications of the THz-wave in spectrum analysis [1, 2], biology and medicine [3, 4], communications [5], security technologies [6] and quality control [7] have raised much interest in terahertz photonics. Unfortunately, lack of practical terahertz sources restricts the applications of the THz-wave. Until there are more practical sources available, the full potential of terahertz radiation will remain, to a great extent, unrealized. Due to the interest in exploiting this region there are many schemes proposed on source technologies over the last fifteen years or so. [8-11] Among many electronic and optical methods for the THz-wave generation, the THz-wave parametric oscillator (TPO) exhibits many advantages, such as compactness, narrow linewidth, coherent, wide tunable range, high-power output and room temperature operation [9]. For efficient generation of the THz-wave, $\mathrm{MgO}: \mathrm{LiNbO}_{3}$ is one of the most suitable crystals due to its large nonlinear coefficient and its wide transparency range. [12] In the TPO both noncollinear phasematching configuration and quasi-phase-matching configuration can perform well. The tuned THz-wave can be realized by varying phase-matching angle, pump wavelength and operation temperature.

In this letter, the frequency tuning characteristics of the THz-wave by changing the pump wavelength, the phasematching angle, the operation temperature and the poling period of the PPLN crystal are investigated. Parametric gain coefficients of the THz-wave under different working temperatures are analyzed.

\section{PHASE-MATCHING SCHEMES}

The phase-matching in the TPO is necessary to avoid destructive interference of the Stokes wave and the THz-wave which are produced by the stimulated Raman scattering. For a $\mathrm{LiNbO}_{3}$ crystal the refractive index in the terahertz range is around 5, as compared to 2 in the near infrared, so birefringence phase-matching is not applicable in this case. One method that has been used by several groups is noncollinear phase-matching based on the bulk $\mathrm{LiNbO}_{3}$ crystal as the nonlinear gain medium, in which the pump wave, the Stokes wave and the THz-wave are

\footnotetext{
*Corresponding author:1zy8376@yahoo.com.cn

Color versions of one or more of the figures in this paper are available online.
} 
all non-parallel with each other, as is shown in Fig. 1(a). For the THz-wave parametric process, two requirements have to be fulfilled: the energy conservation condition

$$
\omega_{\mathrm{p}}=\omega_{\mathrm{s}}+\omega_{\mathrm{T}}
$$

and the phase-matching condition

$$
\vec{k}_{\mathrm{p}}=\vec{k}_{\mathrm{s}}+\vec{k}_{\mathrm{T}}
$$

Here, $\omega_{\mathrm{p}}, \omega_{\mathrm{s}}, \omega_{\mathrm{\Gamma}}$ are the angular frequencies while $\vec{k}_{p}$, $\vec{k}_{s}, \vec{k}_{T}$ are the wave-vectors of the pump, the Stokes and the $\mathrm{THz}$ wave, respectively. The phase-matching condition can be rewritten as

$$
k_{\mathrm{T}}^{2}=k_{\mathrm{p}}^{2}+k_{\mathrm{s}}^{2}-2 k_{\mathrm{p}} k_{\mathrm{s}} \cos \theta
$$

where $\theta$ is the angle between the pump wave and the Stokes wave.

Usually, collinear phase-matching is the preferred configuration for a nonlinear frequency conversion process because it provides the longest interaction length. In recent years PPLN has been widely investigated for generating the $\mathrm{THz}$ radiation, which ensures two or even three mixing waves collinearly propagate, as is shown in Fig. 1 (b-e). In quasi-phase-matching configuration, the phase-matching condition

$$
\vec{k}_{\mathrm{p}}=\vec{k}_{\mathrm{s}}+\vec{k}_{\mathrm{T}}+\vec{k}_{\Lambda}
$$

has to be fulfilled, where $\vec{k}_{\Lambda}$ is the grating vector of an alternating second-order nonlinearity induced by periodic poling of crystal. In the Fig. 1(b) the forwards parametric terahertz process is achieved by $\vec{k}_{A}$ being antiparallel to the pump $\vec{k}_{p}$, the Stokes $\vec{k}_{s}$ and the THz-wave $\vec{k}_{T}$ wave-vectors. The backward parametric terahertz process is achieved by $\vec{k}_{T}$ travelling backward with respect to the pump $\vec{k}_{p}$ and the Stokes $\vec{k}_{s}$, as is shown in Fig. 1(c). Most of the THz energy generated in the forwards and the backwards process is absorbed by the crystal due to the large absorption coefficients in the terahertz range. In Fig. 1(d), the grating vector $\vec{k}_{\Lambda}$ is arranged perpendicular to the pump wave propagation direction, thereby allowing parallel propagation of the pump and the Stokes waves while still retaining the rapid exiting of the THz-wave through the side facet of the crystal. The generated THz-wave is extracted from the nonlinear crystal by an array of high resistivity Si-prisms avoiding total internal reflection. In Fig. 1(e), the pump and the Stokes wave are collinear, while the THz-wave propagates perpendicular to the side facet of the crystal. The THz-wave is coupled out without any coupler, so the loss is low and the beam quality is high.

(a)

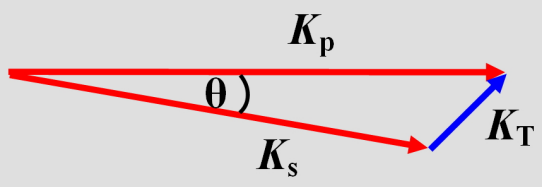

(b)

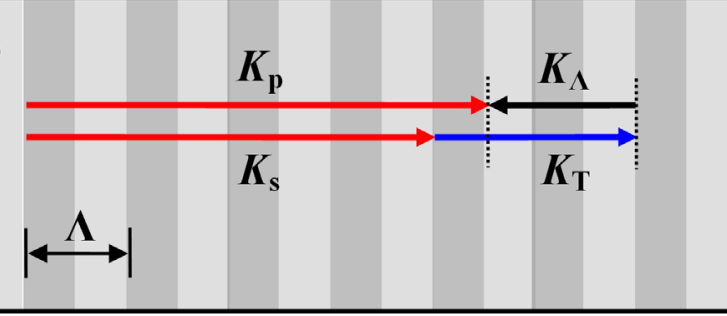

(c)
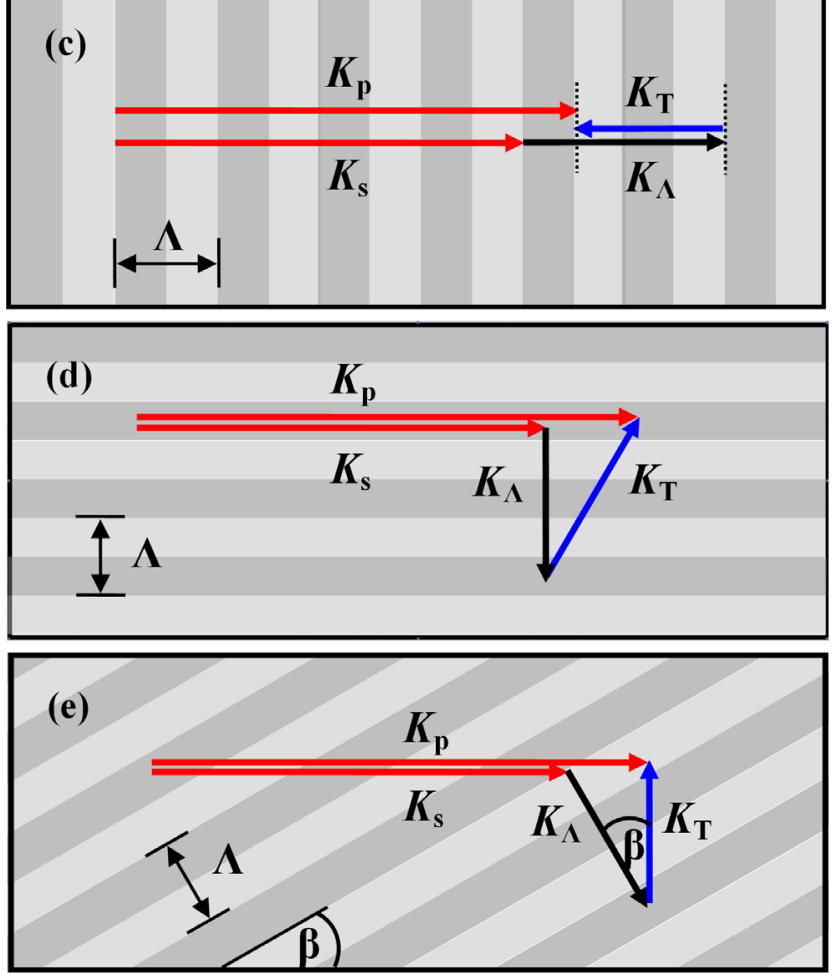

FIG. 1. Phase-matching schemes. (a) Noncollinear phasematching. (b) Quasi-phase-matching, grating vector parallel to the pump wave propagation and the THz-wave propagation direction along with pump wave propagation. (c) Quasi-phasematching, grating vector parallel to the pump wave propagation and the THz-wave travelling backwards with respect to the pump wave propagation. (d) Quasi-phase-matching scheme with grating vector perpendicular to the pump wave propagation. (e) Slant-stripe periodic poling for quasi-phasematching, the THz-wave propagation direction perpendicular to the pump wave propagation. 


\section{TUNING CHARACTERISTICS OF THE THZ-WAVE}

Optical parametric oscillators are more versatile because of their tuning properties. In this section we analyze the tuning characteristics of the THz-wave based on the noncollinear phase-matching and the quasi-phase-matching configuration. According to the Eqs. (1) and (3), the tunable THz-wave frequency vT can be realized by varying the pump wavelength $\lambda_{\mathrm{p}}$ and the phase-matching angle $\theta$. Such tuning is shown in Fig. 2. The THz-wave frequency $v_{\mathrm{T}}$ is sensitive to the angle $\theta$, so the rapid tuning can be reached by changing the angle $\theta$. Different from the methods provided by other groups by rotating the gain medium or mirrors, $[9,13]$ here we propose a method for realizing the tuning output of the THz-wave for the first time. A symmetric resonant cavity of the Stokes wave with a diamond configuration is proposed, as is shown in Fig. 3. The angle $\theta$ between the pump wave and the Stokes wave is tuned by moving the cavity mirror $\mathrm{M}_{3}$ backwards and forwards, as a result, the tuned THz-wave can be reached. The incidence angle of the pump wave $\theta_{0}$

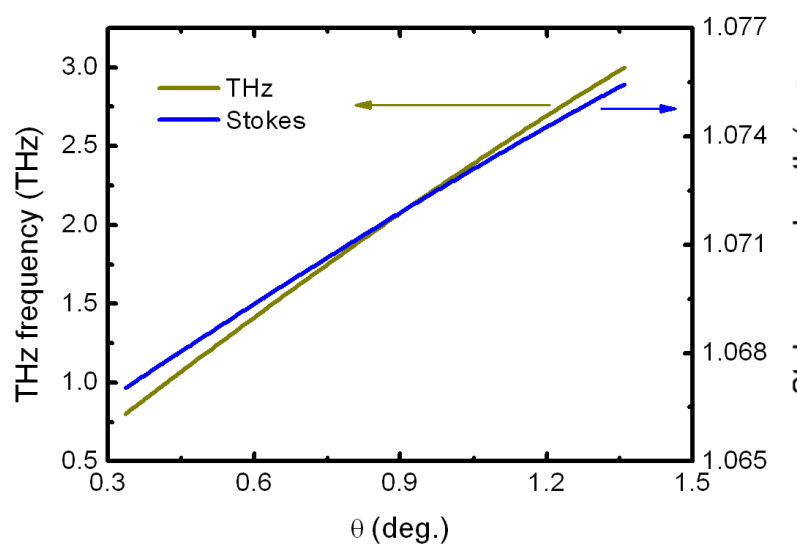

FIG. 2. THz-wave frequency vT versus the phase-matching angle $\theta$ and the Stokes wavelength $\lambda_{\mathrm{s}}$ at room temperature, $\lambda_{\mathrm{p}}=1064 \mathrm{~nm}$.

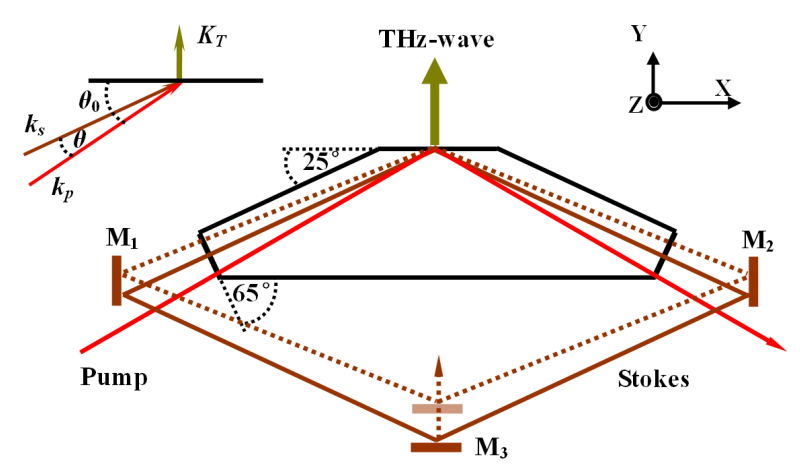

FIG. 3. A symmetric resonant cavity of the Stokes wave with a diamond configuration. The cavity mirror $\mathrm{M}_{3}$ can move backwards and forwards to acquire the tuning angle $\theta$. is set to ensure that the THz-wave with the frequency of $1.5 \mathrm{THz}$ emits perpendicularly from the $\mathrm{LiNbO}_{3}$ crystal. The relationship between the initial position of the mirror M3 and the exit point of the THz-wave $L$ is

$$
L=2 R \tan \theta_{0}
$$

Where $R$ is half the distance between the mirror $\mathrm{M}_{1}$ and $\mathrm{M}_{2}$. The relationship between the movement distance $\Delta L$ of the mirror $\mathrm{M}_{3}$ and the phase-matching angle $\theta$ is

$$
\Delta L=2\left[R \tan \theta_{0}-R \tan \left(\theta_{0}-\theta\right)\right]
$$

According to the Eqs. (3) and (6) the tuning THz-wave can be realized by moving the mirror M3 backwards and forwards. Such tuning is shown in Fig. 4. The tuning range of $0.8-3 \mathrm{THz}$ can be obtained by moving the M3 forwards from 0.87 to $3.51 \mathrm{~mm}$. The method is simple and practical for the tuning output of the THz-wave.

In the process of the THz-wave generation, the THz-wave parametric gain is of vital importance. According to the Ref. (14), the analytical expressions of the exponential gain for the THz-wave can be written as

$$
\begin{aligned}
& g_{\mathrm{T}}=g_{\mathrm{s}} \cos \varphi=\frac{\alpha_{\mathrm{T}}}{2}\left\{\left[1+16 \cos \varphi\left(\frac{g_{0}}{\alpha_{\mathrm{T}}}\right)^{2}\right]^{\frac{1}{2}}-1\right\} \\
& g_{0}^{2}=\frac{\omega_{\mathrm{s}} \omega_{\mathrm{T}}}{128 \pi^{2} \varepsilon_{0} c^{3} n_{\mathrm{s}} n_{\mathrm{T}} n_{\mathrm{p}}} I_{\mathrm{p}}\left(d_{\mathrm{E}}^{\prime}+\sum_{j} \frac{S_{j} \omega_{0_{j}}^{2} d^{\prime}{ }_{\mathrm{Q}_{j}}}{\omega_{0_{j}}^{2}-\omega_{\mathrm{T}}^{2}}\right)^{2} \\
& \alpha_{\mathrm{T}}=2 \frac{\omega_{\mathrm{T}}}{c} \operatorname{Im}\left(\varepsilon_{\infty}+\sum_{j} \frac{S_{j} \omega_{0_{j}}^{2}}{\omega_{0_{j}}^{2}-\omega_{\mathrm{T}}^{2}-i \omega_{\mathrm{T}} \Gamma_{j}}\right)^{\frac{1}{2}}
\end{aligned}
$$

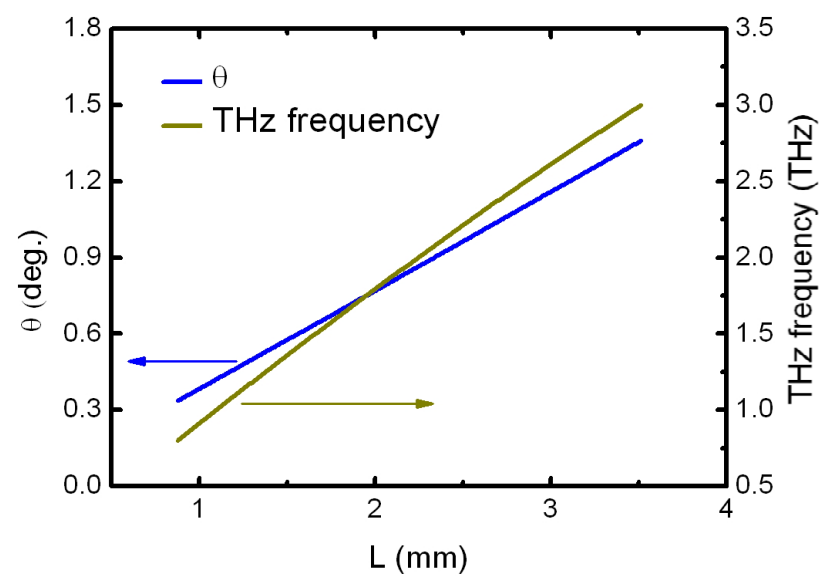

FIG. 4. The movement distance $\Delta L$ of the mirror $\mathrm{M}_{3}$ versus the angle $\theta$ and the THz-wave frequency $v_{\mathrm{T}}, \mathrm{R}=60 \mathrm{~mm}, \theta_{0}=$ $63.53^{\circ}$. 
where $\varphi$ is the phase-matching angle between the THz-wave and the pump wave, $\omega_{0 j}$ and $S_{j}$ are the eigenfrequency and the oscillator strength of the lowest $A_{1}$-symmetry phonon mode, respectively. $I_{\mathrm{p}}$ is the pump power density, $g_{\mathrm{s}}$ is the gain coefficient of the Stokes wave. $n_{\mathrm{p}}, n_{\mathrm{s}}$ and $n_{\mathrm{T}}$ are the refractive indices of the pump wave, the Stokes wave and the THz-wave, respectively. $d_{E}^{\prime}$ and $d_{Q}^{\prime}$ are related to the second-order and third-order nonlinear parametric processes, respectively. The values of parameters of Eqs. (7)-(9) are presented in Ref. (14). Fig. 5 shows the relationship between the THz-wave parametric gain coefficient gT and the angle $\theta$ as $I_{\mathrm{p}}$ equals to 60,100 and $150 \mathrm{MW} / \mathrm{cm}^{2}$, respectively. From the figure we find the $\mathrm{gT}$ increases rapidly to the peak, and then decreases slowly to the lower values. The maximum values of the tuning curves move to the high frequency band as the $I_{\mathrm{p}}$ changes from 60 to 100 and 150 $\mathrm{MW} / \mathrm{cm}^{2}$.

The tuned THz-wave can be achieved also by varying the pump wavelength $\lambda_{\mathrm{p}}$. Fig. 6 shows the relationship between the THz-wave frequency $v_{\mathrm{T}}$ and the pump wave-

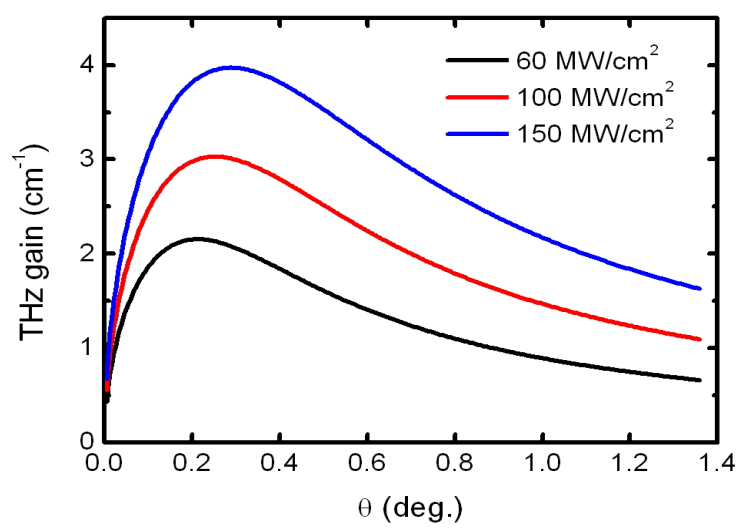

FIG. 5. The parametric gain coefficient $g_{\mathrm{T}}$ versus the angle $\theta$ at room temperature, $\lambda_{\mathrm{p}}=1064 \mathrm{~nm}, I_{\mathrm{p}}=60,100$ and 150 $\mathrm{MW} / \mathrm{cm}^{2}$, respectively.

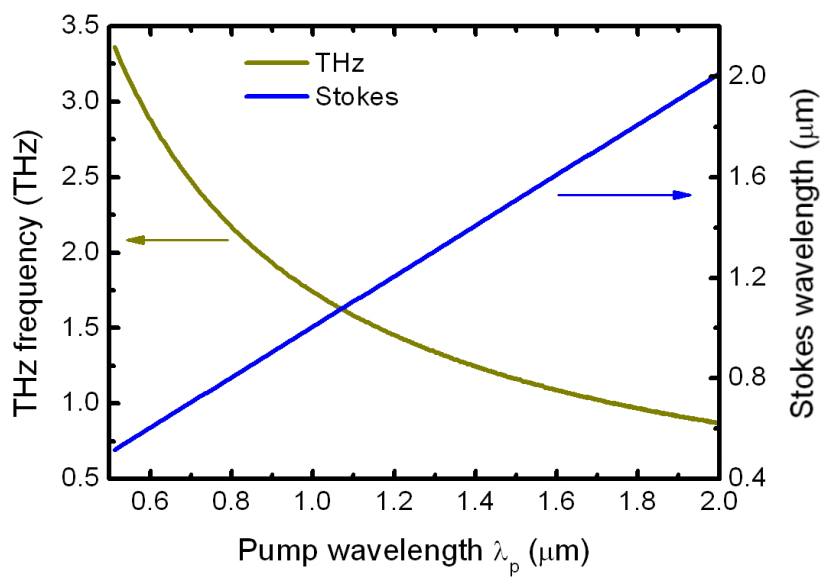

FIG. 6. The THz-wave frequency $v_{\mathrm{T}}$ versus the pump wavelength $\lambda_{\mathrm{p}}$ and the Stokes wavelength $\lambda_{\mathrm{s}}$ at room temperature, $\theta=0.7^{\circ}$. length $\lambda_{\mathrm{p}}$ at room temperature. The $v_{\mathrm{T}}$ decreases rapidly and slowly with the increase of the pump wavelength $\lambda_{\mathrm{p}}$. The pump wavelength not only varies the THz-wave frequency, but also affects the parametric gain of the THz-wave. Fig. 7 shows the parametric gain coefficient gT with the changing of the pump wavelength $\lambda_{\mathrm{p}}$. As the $\lambda_{\mathrm{p}}$ changes from 0.5 to $4 \mu \mathrm{m}$, the $g_{\mathrm{T}}$ increases rapidly to the peak, and then decreases slowly to the lower values. From the figure we find that the maximum values of the tuning curves move to the lower wavelength band as the $I_{\mathrm{p}}$ changes from 60 to 100 and $150 \mathrm{MW} / \mathrm{cm}^{2}$.

The tuning THz-wave can be achieved by changing the working temperature of the $\mathrm{LiNbO}_{3}$ crystal. The relationship among the crystal temperature, the THz-wave frequency vT and the Stokes wavelength $\lambda_{\mathrm{p}}$ is shown in Fig. 8 . Temperature dependence of the refractive index of the $\mathrm{LiNbO}_{3}$ crystal in the terahertz range is reported in Ref. (12). As the temperature varies from $40^{\circ} \mathrm{C}$ to $200^{\circ} \mathrm{C}$, the THz-wave in the range of 1.81-1.84 THz can be obtained. Compared with the tuning characteristics by varying the

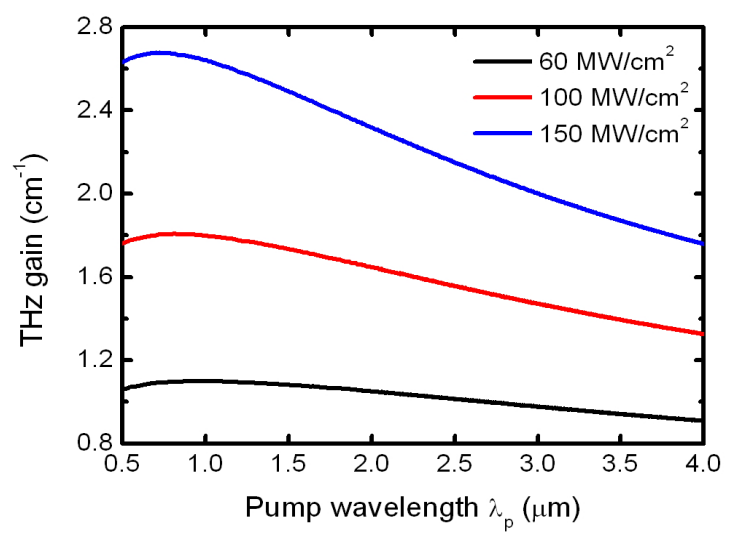

FIG. 7. The parametric gain coefficient $g_{\mathrm{T}}$ versus the pump wavelength $\lambda_{\mathrm{p}}$ at room temperature, $\theta=0.7 \mathrm{o}, I_{\mathrm{p}}=60,100$ and $150 \mathrm{MW} / \mathrm{cm}^{2}$, respectively.

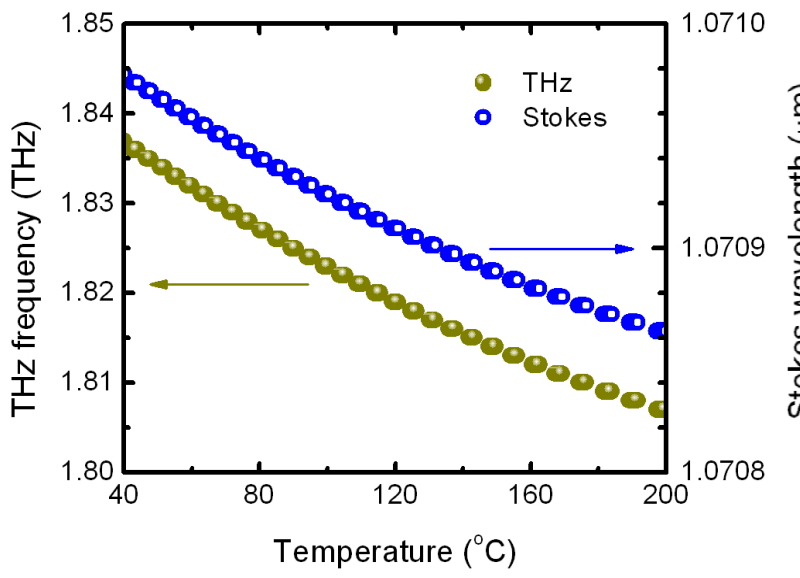

FIG. 8. The temperature tuning characteristics of the THz-wave, $\lambda_{\mathrm{p}}=1064 \mathrm{~nm}, \theta=0.7^{\circ}$. 


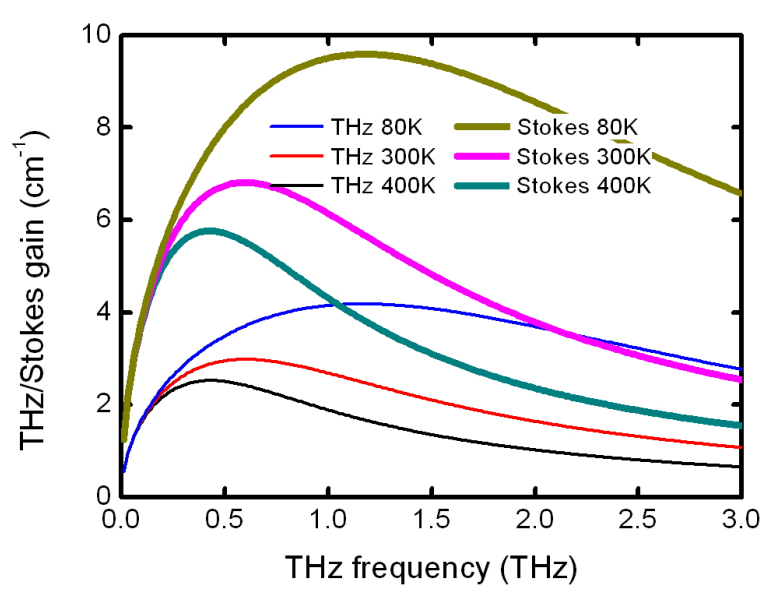

FIG. 9. Gain coefficients of the THz-wave and the Stokes wave, $\lambda_{\mathrm{p}}=1064 \mathrm{~nm}, I_{\mathrm{p}}=100 \mathrm{MW} / \mathrm{cm}^{2}$.

phase-matching and the pump wavelength, the THz-wave frequency is insensitive to the working temperature. The crystal temperature not only affects the phase-matching condition, but also has a significant impact on the parametric gain coefficient $g_{\mathrm{T}}$ and $g_{\mathrm{s}}$. The characteristics of $g_{\mathrm{T}}$ and $g_{\mathrm{s}}$ at different temperatures are shown in Fig. 9. From the figure we find that the $g_{\mathrm{T}}$ and $g_{\mathrm{s}}$ increase along with the decrease of the temperature. The damping coefficient of the lowest $A_{1}$-symmetry phonon mode in the $\mathrm{LiNbO}_{3}$ crystal reduces with the decrease of the temperature[15], resulting in the enlargement of the parametric gain. As discussed above, the enhanced output of the THz-wave can be realized by reducing the working temperature.

The tuning output of the THz-wave can be realized in quasi-phase-matching configuration by varying the poling period of the PPLN crystal and the phase-matching angle. In this section we analyze the tuning characteristics based on the model shown in Fig. 1(e), since the THz-wave is coupled out perpendicularly to the side surface of the PPLN crystal without using any output coupler. According to the Fig. 1(e), the poling period $\Lambda$ and the phase-matching angle $\beta$ between the THz-wave propagation direction and the grating vector are

$$
\begin{aligned}
& \Lambda=\left[\left(\frac{n_{\mathrm{T}}}{\lambda_{\mathrm{T}}}\right)^{2}+\left(\frac{\mathrm{n}_{\mathrm{p}}}{\lambda_{\mathrm{p}}}-n_{\mathrm{s}}\left(\frac{1}{\lambda_{\mathrm{p}}}-\frac{1}{\lambda_{\mathrm{T}}}\right)\right)^{2}\right]^{-\frac{1}{2}} \\
& \sin \beta=\Lambda\left[\frac{\mathrm{n}_{\mathrm{p}}}{\lambda_{\mathrm{p}}}-n_{\mathrm{s}}\left(\frac{1}{\lambda_{\mathrm{p}}}-\frac{1}{\lambda_{\mathrm{T}}}\right)\right]
\end{aligned}
$$

Where $\lambda_{\mathrm{T}}$ is the wavelength of the THz-wave. The tuning THz-wave versus the phase-matching angle $\beta$ and poling period $\Lambda$ at room temperature is shown in Fig. 10. With the increase of the THz-wave frequency $\mathrm{vT}$, the poling period $\Lambda$ decreases rapidly and then slowly, while the

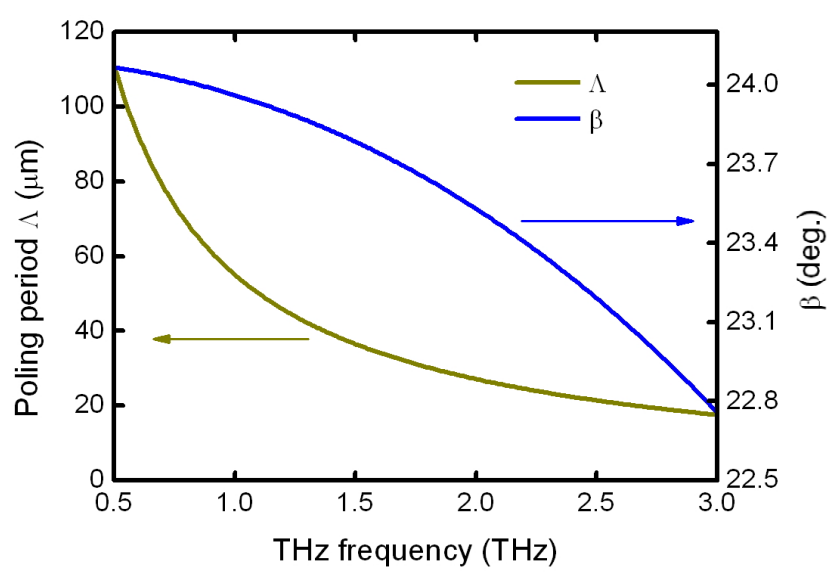

FIG. 10. The THz-wave frequency vT versus the phase-matching angle $\beta$ and poling period $\Lambda$ at room temperature, $\lambda_{\mathrm{p}}=1064$ $\mathrm{nm}$.

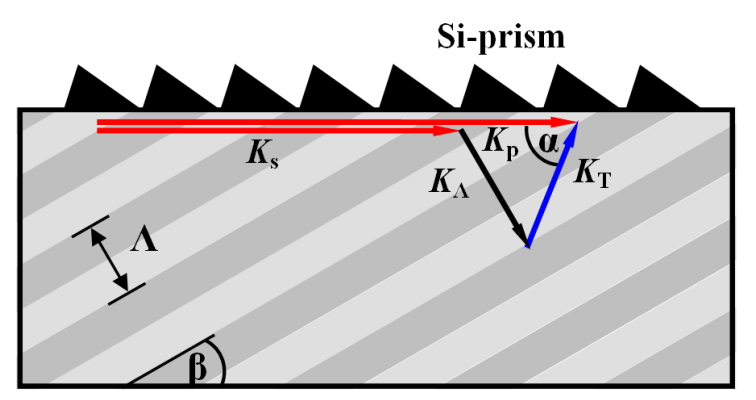

FIG. 11. Schematic diagram of the quasi-phase-matching in PPLN crystal as the THz-wave wave-vector is not perpendicular to the the side surface of the PPLN crystal.

angle $\beta$ decreases slowly and then rapidly. At the point of $1.5 \mathrm{THz}$ where the output of the THz-wave is of the most intensity [16], the poling period $\Lambda$ equals to $36.5 \mu \mathrm{m}$ and the angle $\beta$ equals to $23.8^{\circ}$.

As the THz-wave propagation direction is not perpendicular to the side surface of the PPLN crystal, the THz-wave can be coupled out by employing an array of Si-prisms to avoid total internal reflection [16, 17], as is shown in Fig. 11. The angle $a$ is between the THz-wave wave-vector and the pump wave wave-vector. The relationship between the angle $a$ and the THz-wave wavelength $\lambda_{\mathrm{T}}$ is

$$
\sin \alpha=\frac{\lambda_{\mathrm{T}} \cos \beta}{n_{\mathrm{T}} \Lambda}
$$

According to the Eqs. (1) and (12), the tuning THz-wave with different propagation directions can be realized in a PPLN crystal with a fixed poling period $\Lambda$ and a fixed angle $\beta$ by employing a tuning Stokes seed beam. Such tuning is shown in Fig. 12, assuming the poling period $\Lambda$ of $36.5 \mu \mathrm{m}$ and the angle $\beta$ of $23.8^{\circ}$ where the THz-wave output is the most intense. Since the refractive indexes of the $\mathrm{THz}$-wave in $\mathrm{LiNbO}_{3}$ crystal and high-resistivity $\mathrm{Si}$ are 


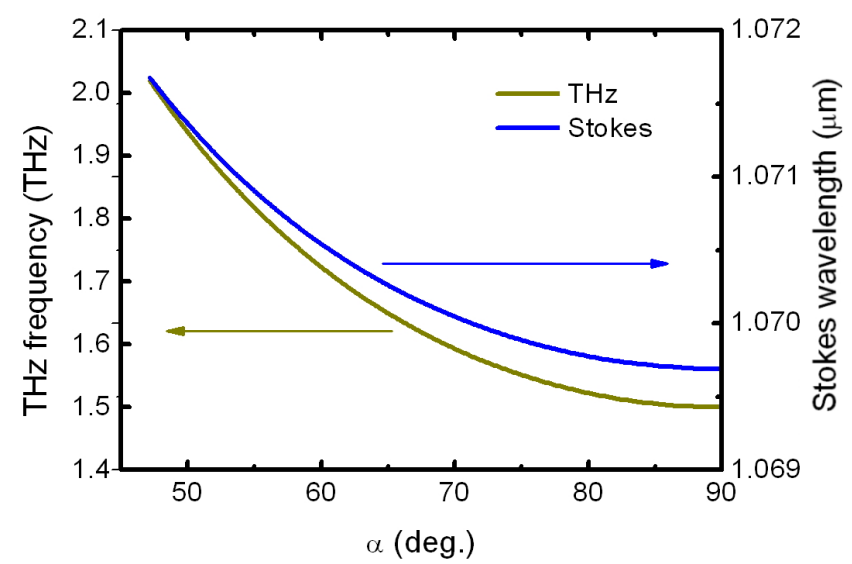

FIG. 12. The THz-wave frequency vT versus the angle $\alpha$ and the Stokes wavelength $\lambda_{\mathrm{s}}$ at room temperature, $\Lambda=36.5 \mu \mathrm{m}$, $\beta=23.80, \lambda_{\mathrm{p}}=1064 \mathrm{~nm}$.

approximately 5.1 and 3.4 respectively, the minimum value of the angle $\alpha$ is $47.2^{\circ}$ to avoid total internal reflection of the THz-wave. From the figure we find that by injecting the tuning Stokes seed beam in the range of 1069.7-1071.7 $\mathrm{nm}$ we can obtain the tuning THz-wave from 1.5 to 2.02 $\mathrm{THz}$. The analysis here provides a choice for the tuning THz-wave by employing a PPLN crystal with a fixed poling period $\Lambda$ and a fixed angle $\beta$.

\section{CONCLUSION}

The THz-wave tuning characteristics of the noncollinear phase-matching TPO and the quasi-phase-matching TPO are investigated. In the condition of the noncollinear phase-matching configuration, the $\mathrm{THz}$-wave frequency is sensitive to the variation of the phase-matching angle $\theta$ and the pump wavelength $\lambda_{\mathrm{p}}$, while insensitive to the variation of the crystal temperature. The phase-matching angle $\theta$, the pump wavelength $\lambda_{\mathrm{p}}$ and the crystal temperature affect the parametric gain coefficients of the THz-wave. The tuning THz-wave can be realized in quasi-phase-matching configuration by varying the poling period of the PPLN crystal and the phase-matching angle. Employing the PPLN crystal with the poling period $\Lambda$ of $36.5 \mu \mathrm{m}$ and the angle $\beta$ of $23.8^{\circ}$, we can obtain the tuning THz-wave from 1.5 to $2.02 \mathrm{THz}$ by injecting the Stokes seed beam with the wavelength $\lambda_{s}$ in the range of 1069.7-1071.7 nm.

\section{ACKNOWLEDGMENT}

This work was supported by the National Natural Science Foundation of China (Grant Nos. 61201101 and 61172010).

\section{REFERENCES}

1. Y. Kim, K. H. Jin, J. C. Ye, J. Ahn, and D. Yee, "Wavelet power spectrum estimation for high-resolution terahertz time-domain spectroscopy,” J. Opt. Soc. Korea 15, 103-108 (2011).

2. C. Kang, C. Kee, I. Sohn, and J. Lee, "Spectral properties of THz-periodic metallic structures," J. Opt. Soc. Korea 12, 196-199 (2008)

3. Y. Y. Wang, H. Minamide, M. Tang, T. Notake, and H. Ito, "Study of water concentration measurement in thin tissues with terahertz-wave parametric source," Opt. Express 18, 15504-15512 (2010).

4. E. Jung, M. Lim, K. Moon, Y. Do, S. Lee, H. Han, H. Choi, K. Cho, and K. Kim, "Terahertz pulse imaging of micro-metastatic lymph nodes in early-stage cervical cancer patients," J. Opt. Soc. Korea 15, 155-160 (2011).

5. T. Kleine-Ostmann and T. Nagatsuma, "A review on terahertz communications research," J. Infrared Milli. Terahz. Waves 32, 143-171 (2011).

6. H. B. Liu, H. Zhong, N. Karpowicz, Y. Chen, and X. C. Zhang, "Terahertz spectroscopy and imaging for defense and security applications," Proc. IEEE 95, 1514-1527 (2007).

7. F. Rutz, M. Koch, S. Khare, M. Moneke, H. Richter, and U. Ewert, "Terahertz quality control of polymeric products," Int. J. Infrared Millimeter Waves 27, 547-556 (2006).

8. C. Kang, Y. L. Lee, C. Jung, H. K. Yoo, and C. Kee, "Effects of uncertain phase-matching wave vectors of rotating fan-out type poled $\mathrm{LiNbO}_{3}$ on $\mathrm{THz}$ generation," Opt. Express 18, 21484-21489 (2010).

9. K. Kawase, J. Shikata, and H. Ito, "Terahertz wave parametric source,” J. Phys. D: Appl. Phys. 34, R1-R14 (2001).

10. J. Kiessling, F. Fuchs, K. Buse, and I. Breunig, "Pumpenhanced optical parametric oscillator generating continuous wave tunable terahertz radiation," Opt. Lett. 36, 4374-4376 (2011).

11. D. Molter, M. Theuer, and R. Beigang, "Nanosecond terahertz optical parametric oscillator with a novel quasi-phase-matching scheme in lithium niobate," Opt. Express 17, 6623-6628 (2009).

12. R. Sowade, Breunig, C. Tulea, and K. Buse, "Nonlinear coefficient and temperature dependence of the refractive index of lithium niobate crystals in the terahertz regime," Appl. Phys. B 99, 63-66 (2010).

13. H. Minamide, T. Ikari, and H. Ito, "Frequency-agile terahertzwave parametric oscillator in a ring-cavity configuration," Rev. Sci. Instrum. 80, 123104 (2009).

14. S. S. Sussman, "Tunable light scattering from transverse optical modes in lithium niobate," Report of Microwave Lab, Stanford University No. 1851, 22-34 (1970).

15. W. D. Johnston Jr. and I. P. Kaminow, "Temperature dependence of Raman and Rayleigh scattering in $\mathrm{LiNbO}_{3}$ and $\mathrm{LiTaO}_{3}$," Phys. Rev. 168, 1045-1054 (1968).

16. T. Ikari, X. B. Zhang, H. Minamide, and H. Ito, "THz-wave parametric oscillator with a surface-emitted configuration," Opt. Express 14, 1604-1610 (2006).

17. K. Kawase, J. Shikata, H. Minamide, K. Imai, and H. Ito, "Arrayed silicon prism coupler for a terahertz-wave parametric oscillator," Appl. Opt. 40, 1423-1426 (2001). 